Frohn, Julia; Rödel, Laura

\title{
Anschlussstellen zwischen Sprachbildung und adaptiver Lehrkompetenz für den inklusiven Unterricht
}

Brodesser, Ellen [Hrsg.]; Frohn, Julia [Hrsg.]; Welskop, Nena [Hrsg.]; Liebsch, Ann-Catherine [Hrsg.]; Moser, Vera [Hrsg.]; Pech, Detlef [Hrsg.]: Inklusionsorientierte Lehr-Lern-Bausteine für die Hochschullehre. Ein Konzept zur Professionalisierung zukünftiger Lehrkräfte. Bad Heilbrunn : Verlag Julius Klinkhardt 2020, S. 37-44. (Interdisziplinäre Beiträge zur Inklusionsforschung)

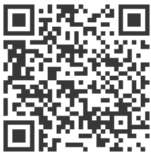

Quellenangabe/ Reference:

Frohn, Julia; Rödel, Laura: Anschlussstellen zwischen Sprachbildung und adaptiver Lehrkompetenz für den inklusiven Unterricht - In: Brodesser, Ellen [Hrsg.]; Frohn, Julia [Hrsg.]; Welskop, Nena [Hrsg.]; Liebsch, Ann-Catherine [Hrsg.]; Moser, Vera [Hrsg.]; Pech, Detlef [Hrsg.]: Inklusionsorientierte Lehr-Lern-Bausteine für die Hochschullehre. Ein Konzept zur Professionalisierung zukünftiger Lehrkräfte. Bad Heilbrunn : Verlag Julius Klinkhardt 2020, S. 37-44 - URN:

urn:nbn:de:0111-pedocs-190155 - DOI: 10.25656/01:19015

https://nbn-resolving.org/urn:nbn:de:0111-pedocs-190155

https://doi.org/10.25656/01:19015

in Kooperation mit / in cooperation with:

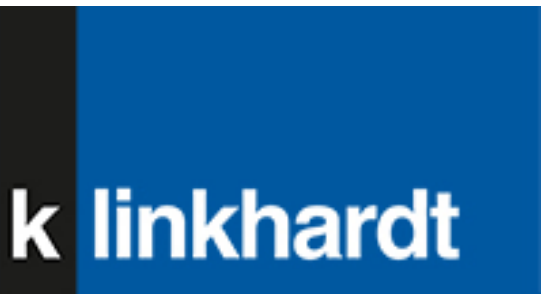

http://www.klinkhardt.de

\section{Nutzungsbedingungen}

Dieses Dokument steht unter folgender Creative Commons-Lizenz: http://creativecommons.org/licenses/by-nc-sa/4.0/deed.de - Sie dürfen das Werk bzw. den Inhalt unter folgenden Bedingungen vervielfältigen, verbreiten und öffentlich zugänglich machen sowie Abwandlungen und Bearbeitungen des Werkes bzw. Inhaltes anfertigen: Sie müssen den Namen des Autors/Rechteinhabers in der von ihm festgelegten Weise nennen. Dieses Werk bzw. der Inhalt darf nicht für kommerzielle Zwecke verwendet werden. Die neu entstandenen Werke bzw. Inhalte dürfen nur unter Verwendung von Dizenzbedingungen weitergegeben werden, die mit denen dieses Lizenzbedingungen weitergegeben werden,

Mit der Verwendung dieses Dokuments erkennen Sie die Nutzungsbedingungen an.

\section{Terms of use}

This document is published under following Creative Commons-License: http://creativecommons.org/licenses/by-nc-sa/4.0/deed.en - You may copy, distribute and transmit, adapt or exhibit the work in the public and alter, transform or change this work as long as you attribute the work in the manner specified by the author or licensor. You are not allowed to make commercial use of the the author or licensor. You are not allowed to make commercial may distribute the resulting work only under this or a comparable license.

By using this particular document, you accept the above-stated conditions of

\section{Kontakt / Contact:}

\section{peDOcs}

DIPF | Leibniz-Institut für Bildungsforschung und Bildungsinformation Informationszentrum (IZ) Bildung

E-Mail: pedocs@dipf.de

Internet: www.pedocs.de

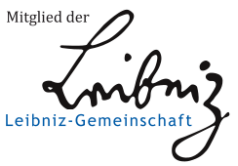


Interdisziplinäre Beiträge zur Inklusionsforschung

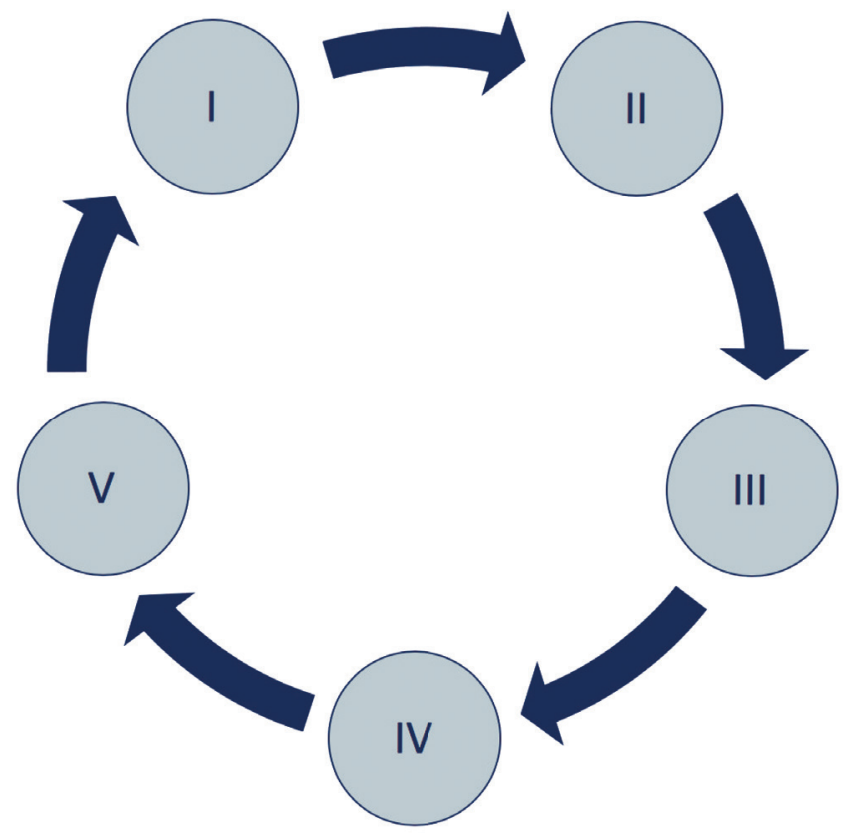

Ellen Brodesser / Julia Frohn /

Nena Welskop / Ann-Catherine Liebsch /

Vera Moser / Detlef Pech

(Hrsg.)

\section{Inklusionsorientierte Lehr-Lern- Bausteine für die Hochschullehre}

Ein Konzept zur Professionalisierung zukünftiger Lehrkräfte 
Brodesser / Frohn / Welskop / Liebsch / Moser / Pech Inklusionsorientierte Lehr-Lern-Bausteine für die Hochschullehre 
Interdisziplinäre Beiträge zur Inklusionsforschung

herausgegeben von Marina Egger, Jullia Frohn,

Vera Moser und Detlef Pech 


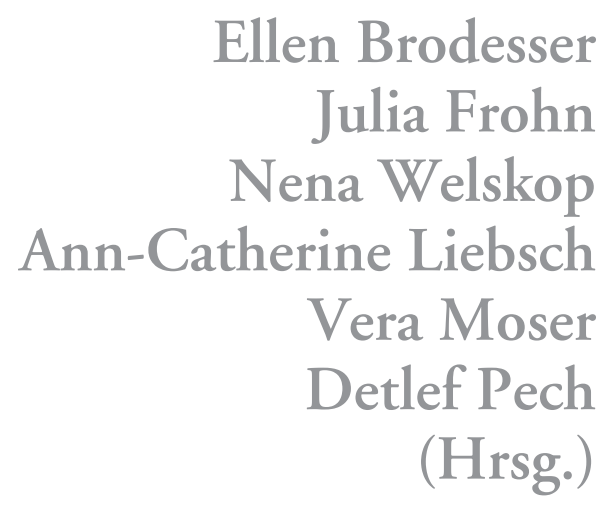

\section{Inklusionsorientierte Lehr-Lern- Bausteine für die Hochschullehre}

Ein Konzept zur Professionalisierung zukünftiger Lehrkräfte 
Das diesem Buch zugrundeliegende Vorhaben wurde im Rahmen der gemeinsamen „Qualitätsoffensive Lehrerbildung“ von Bund und Ländern mit Mitteln des Bundesministeriums für Bildung und Forschung unter dem Förderkennzeichen 01JA1620 gefördert.

Die Verantwortung für den Inhalt der Veröffentlichung liegt bei den Autor*innen.

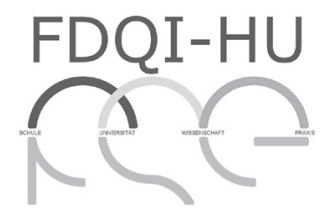

GEFÖRDERT VOM

Bundesministerium
für Bildung
und Forschung

Dieser Titel wurde in das Programm des Verlages mittels eines Peer-Review-Verfahrens aufgenommen.

Für weitere Informationen siehe www.klinkhardt.de.

Bibliografische Information der Deutschen Nationalbibliothek

Die Deutsche Nationalbibliothek verzeichnet diese Publikation in der Deutschen Nationalbibliografie; detaillierte bibliografische Daten

sind im Internet abrufbar über http://dnb.d-nb.de.

2020.i. (c) by Julius Klinkhardt.

Satz und Grafik Umschlagseite 1: Kay Fretwurst, Spreeau.

Druck und Bindung: AZ Druck und Datentechnik, Kempten.

Printed in Germany 2020.

Gedruckt auf chlorfrei gebleichtem alterungsbeständigem Papier.

(c)(i)(5) Die Publikation (mit Ausnahme aller Fotos, Grafiken und Abbildungen) ist veröffentlicht unter der Creative Commons-Lizenz: CC BY-NC-SA 4.0 International

https://creativecommons.org/licenses/by-nc-sa/4.0/

ISBN 978-3-7815-5798-7 Digital

doi.org/10.35468/5798

ISBN 978-3-7815-2361-6 Print 


\section{Inhalt}

1 Einführung: Inklusionsorientierte Lehr-Lern-Bausteine für die Hochschullehre eine Begründung durch Praxisbezug, Theorie und Methodik ............... Ellen Brodesser, Julia Frohn, Nena Welskop, Ann-Catherine Liebsch, Vera Moser und Detlef Pech

2 Heterogenitätssensibilität, adaptive Lehrkompetenz und Sprachbildung als Ausgangspunkte für die Entwicklung

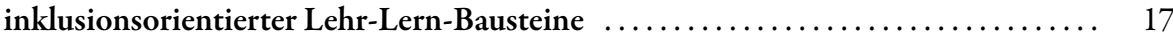

2.1 Heterogenitätssensibilität als Voraussetzung adaptiver Lehrkompetenz ....... 19 Nena Welskop und Vera Moser

2.2 Lehrkräfteprofessionalisierung: adaptive Lehrkompetenz für inklusiven Unterricht Julia Frohn, Lena Schmitz und Hans Anand Pant

2.3 Anschlussstellen zwischen Sprachbildung und adaptiver Lehrkompetenz für den inklusiven Unterrich Julia Frohn und Laura Rödel

3 Inklusionsorientierte Lehr-Lern-Bausteine: theoretische Einbettung, didaktische Kommentierung und Einsatz in der inklusionssensiblen Hochschullehre

3.1 Einleitung: Entwicklung und Umsetzung inklusionsorientierter Lehr-Lern-Bausteine Ann-Catherine Liebsch

3.2 Der Baustein Heterogenitätssensibilität: inklusionspädagogische Grundlegung

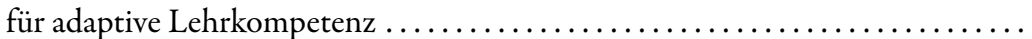
Dietlind Gloystein

3.3 Der Baustein Adaptive diagnostische Kompetenz: ein Selbstversuch und inklusionssensible pädagogische Diagnostik als Impuls für Perspektivwechsel und professionelle Reflexion

Dietlind Gloystein und Julia Frohn

3.4 Der Baustein Adaptive didaktische Kompetenz: inklusive (Fach-)Didaktik und adaptive didaktische Kompetenz .......... 76 Fabian Eckert und Ann-Catherine Liebsch

3.5 Der Baustein Adaptive Klassenführungskompetenz: effektive Klassenführung als Basis für den inklusiven Unterricht ........... Ann-Catherine Liebsch und Yasmin Patzer

3.6 Der Baustein Sprachbildung: ein Lehr-Lern-Angebot für die inklusionssensible fachdidaktische Lehre Laura Rödel 
4 Multiperspektivische Evaluation $\ldots \ldots \ldots \ldots \ldots \ldots \ldots \ldots \ldots \ldots \ldots \ldots \ldots, 111$

4.1 Heterogenitätssensibilität angehender Lehrkräfte: empirische Ergebnisse . .... 113 Lena Schmitz, Toni Simon und Hans Anand Pant

4.2 Adaptive Lehrkompetenz: Bildung von Indizes und empirische Ergebnisse zur Wirkung universitärer Lehrveranstaltungen ...... 124 Lena Schmitz, Ellen Brodesser und Hans Anand Pant

4.3 Den üblichen Weg verlassen. Objektiv-hermeneutische Analyse der Interviews mit Dozierenden zum Einsatz inklusionsorientierter Lehr-Lern-Bausteine in fachdidaktischen Seminaren ................. 13 Ellen Brodesser, Nena Welskop und Julia Frohn

5 Ausblick: Inklusionsorientierung in verschiedenen Phasen der Lehrkräftebildung

5.1 Potenziale inklusionsorientierter Lehr-Lern-Bausteine für die Übertragung auf verschiedene Fachdidaktiken und für die MINT-Fächer . ........... 151 Yasmin Patzer, Julia Frohn und Niels Pinkwart

5.2 Aufbau und Erweiterung von Heterogenitätssensibilität und diagnostischer Kompetenz durch inklusionsorientierte Lehr-Lern-Bausteine in der universitären Lehrkräftebildung: Einordnung und Weiterentwicklung der konzipierten Unterrichtseinheiten aus inklusionspädagogischer Sicht ........ 162 Dietlind Gloystein und Vera Moser

5.3 Impulse aus der Konzeption der inklusionsorientierten Lehr-Lern-Bausteine und aus dem Forschenden Lernen für die Sprachbildung in der ersten Phase der Lehrkräftebildung . . Laura Rödel, Maria Große und Constanze Saunders

5.4 Einsatzpotenziale inklusionsorientierter Lehr-Lern-Bausteine im Vorbereitungsdienst und in der Berliner Mentoringqualifizierung ............ Nena Welskop, Ellen Brodesser und Caroline Körbs

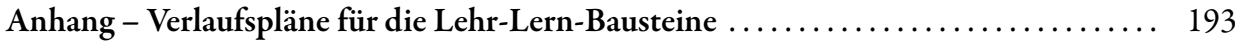

1. Verlaufsplan für den Baustein Heterogenitätssensibilität ................. 194

2. Verlaufsplan für den Baustein Adaptive diagnostische Kompetenz . . . . . . . . . . 195

3. Verlaufsplan für den Baustein Adaptive didaktische Kompetenz . . . . . . . . . . . 197

4. Verlaufsplan für den Baustein Adaptive Klassenführungskompetenz .......... 198

5. Verlaufsplan für den Baustein Sprachbildung ...................... 199

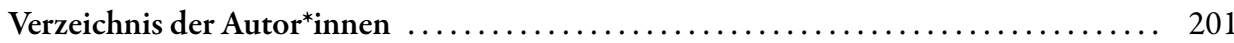




\section{Julia Frohn und Laura Rödel}

\subsection{Anschlussstellen zwischen Sprachbildung und adaptiver Lehrkompetenz für den inklusiven Unterricht}

Die aktuelle Lehrkräftebildung ist von verschiedenen Querschnittsthemen geprägt. Diese bilden sich - je nach Bundesland mit zum Teil unterschiedlichen Bezeichnungen - in unterschiedlich starkem Ausmaß in den Curricula der verschiedenen Phasen ab und/oder sind Gegenstand von Forschungsarbeiten. An der Humboldt-Universität zu Berlin werden zurzeit die Themen Sprachbildung, Inklusion, Medienbildung und Forschendes Lernen fokussiert (vgl. Professional School of Education, 2019). Dabei sind die Bereiche nicht trennscharf voneinander abzugrenzen und weisen mitunter deutliche Synergien auf. Im Rahmen des Projekts Fachdidaktische Qualifizierung Inklusion angehender Lebrkräfte an der Humboldt-Universität zu Berlin (FDQI-HU) werden die beiden Querschnittsthemen Sprachbildung und Inklusion in ihrem Verhältnis zueinander untersucht und bearbeitet, wobei das Ziel der individuellen Kompetenzentwicklung ${ }^{7}$ im Fokus der Entwicklungsarbeit steht. Dieses Ziel kann insbesondere durch adaptives Unterrichten erreicht werden (vgl. Einleitung in diesem Band), weshalb auch das Konstrukt der adaptiven Lehrkompetenz (Beck et al., 2008) im Projekt FDQI-HU beforscht und in Lehrveranstaltungen gefördert wird. Im Folgenden werden zunächst die Konzepte Inklusion und Sprachbildung anhand ihrer Schnittpunkte und Unterschiede in den Fokus gerückt. Anschließend werden Ziele der Sprachbildung mit denen des adaptiven Lehrens - beruhend auf dem Konstrukt der adaptiven Lehrkompetenz - systematisch zusammengebracht: Obwohl nämlich beide Ansätze zentral für die Lehrkräftebildung im Projekt FDQI-HU sind, wurden sie bisher noch nicht zueinander in Beziehung gesetzt. Dieser Beitrag geht daher möglichen Anschlussstellen zwischen den Konzepten nach, um diese für die inklusionssensible Lehrkräftebildung nutzbar zu machen.

\subsubsection{Sprachbildung und Inklusion: zwei getrennte Diskurse?}

„Aus Deutsch für Ausländer wird Deutsch als Fremdsprache wird Deutsch als Zweitsprache wird Durchgängige Sprachbildung ... wird Inklusion?“" (Settinieri, 2017, S. 306)

Dieser Titel eines Aufsatzes von Julia Settinieri legt nahe, dass sich Querschnittsthemen der Lehrkräftebildung in einem stetigen Prozess der Weiterentwicklung befinden. Den von Settinieri genannten Arbeitsbereichen ist gemein, dass sie auf einen Umgang mit der (sprachlichen) Heterogenität von Lerner*innengruppen zielen. Allerdings liegen ihnen - auf gesellschaftliche und politische Entwicklungen reagierend (vgl. Baumann, 2017) - jeweils unterschiedliche Konzepte zugrunde, was entsprechend mit einer „Verschiebung der Begrifflichkeiten und Erweiterung der Zielgruppe" (ebd., S. 10) einhergeht. Indem Settinieri den Begriff Inklusion an den Schluss ihrer Frage stellt, verdeutlicht sie, dass die Diskurse um die Querschnittsthemen Inklusion und Sprachbildung seit einiger Zeit nicht mehr (nur) getrennt voneinander geführt werden. Mit der Setzung eines Fragezeichens macht sie indes darauf aufmerksam, dass diese Entwicklung noch keinesfalls als selbstverständlich gelten und hinterfragt werden kann. Es bleibt also zu erörtern, in welchem Verhältnis Inklusion und Sprachbildung stehen und was die beiden Querschnittsthemen (potentiell) verbindet.

7 Die Kompetenzentwicklung bezieht sich dabei nicht ausschließlich auf den Bereich der kognitiven Entwicklung, sondern betrifft auch die motivationale, soziale, ethisch-normative sowie die performative Dimension des Kompetenzbegriffs (vgl. Frohn, 2019). 
Um diese Fragen beantworten zu können, ist zunächst das diesem Beitrag zugrunde liegende Verständnis von Sprachbildung und Inklusion zu klären. Ein weites Verständnis von Inklusion im Kontext Schule, wie es auch vom Projekt FDQI-HU vertreten wird, bezieht sich auf unterschiedliche Heterogenitätsdimensionen und nimmt diese reflexiv und unter Wahrung einer intersektionalen Perspektive in den Blick (vgl. ausführlicher Simon, 2019). Schulische Inklusion zielt diesem Verständnis nach auf die Partizipation aller Schüler*innen, also auf „effektive Einflussnahme, Mitbestimmung und Entscheidungsmacht“ (Flieger, 2017, S. 179). Partizipation wird „über die gestaltende Teilhabe aller Lernenden in Schule und Unterricht ohne Ausschluss, [...] über Aspekte demokratischer Bildung und des Demokratie-Lernens, [...] über die Aktivierung der Schüler*innen und [...] über die Förderung ko-konstruktiver Lehr-LernProzesse“ (Simon \& Pech, 2019, S. 40) beschrieben. Das Konzept Sprachbildung wiederum wird im Kontext von FDQI-HU ebenfalls zielgruppenübergreifend verstanden. Es zielt auf eine positive gesellschaftliche Partizipation aller Lernenden und eine höhere Bildungsgerechtigkeit insgesamt. Diese Ziele sollen zum einen mit einem sprachsensiblen Fachunterricht, der alle Schüler*innen mit einbezieht, verfolgt werden; zum anderen mit einer gesamtsprachlichen Bildung, die die individuelle Mehrsprachigkeit der Lernenden berücksichtigt, indem Erstund Zweitsprachen, (schulische) Fremdsprachen und vorhandene Registerkompetenzen der Schüler*innen miteinbezogen werden (vgl. ausführlich Lütke, 2019; Rödel \& Lütke, 2019).

Dass Inklusion und Sprachbildung in einem besonderen Verhältnis zueinander stehen, wird mit Blick auf Publikationen der letzten Jahre sowohl aus dem Sprachbildungs- als auch aus dem Inklusionsdiskurs deutlich (vgl. exemplarisch Becker-Mrotzek, 2016; Chilla, 2017; Dirim \& Mecheril, 2018; König \& Friederich, 2015; Riemer, 2017). Auch in Forschungsprojekten werden die beiden Themen miteinander verbunden (neben FDQI-HU z.B. im Potsdamer Qualitätsoffensive-Projekt Professionalisierung - Schulpraktische Studien - Inklusion, Arbeitsbereich ,Inklusion und Heterogenität $\left.{ }^{8}\right)$. Dabei variiert jedoch das je zugrunde liegende Verständnis von Inklusion und Sprachbildung, mitunter wird auch die zielgruppenspezifische Perspektive Deutsch als Zweitsprache (DaZ) eingenommen (vgl. z.B. Berkemeier \& Kaltenbacher, 2014; Riemer, 2017). Zudem unterscheiden sich die Publikationen und Forschungsprojekte in der Intensität, in der sie das Verhältnis von Inklusion und Sprachbildung beleuchten (vgl. im Folgenden Rödel \& Simon, 2018; 2019). Das Spektrum reicht von der einfachen (aber wichtigen) Platzierung des Themas Sprachbildung im Gesamtdiskurs Inklusion (vgl. z.B. Daveri \& Weilbrenner, 2013) bis hin zu einer expliziten Auseinandersetzung mit gemeinsamen oder sich unterscheidenden Konzepten (vgl. z.B. Lütke, 2019; Tschernig \& Vo Thi, 2017).

Bezogen auf die Zielgruppe Kinder und Jugendliche mit DaZ wird im Rahmen der Diskussion um Inklusion und Sprachbildung darauf hingewiesen, dass diese Lernenden von einer konsequent inklusiven Schule insofern profitieren, als sie hier in der Regelschule zu einem Abschluss geführt werden (vgl. Bergemann, 2015). Dieses Argument für das Zusammendenken der beiden Querschnittsaufgaben der Lehrkräftebildung ist zunächst ein pragmatisches, impliziert jedoch zugleich

„die Forderung nach einer konsequenten De-Segregation und Nicht-Diskriminierung bzw. -Benachteiligung von Kindern und Jugendlichen mit Deutsch als Zweit-/Zielsprache bzw. Migrationshintergrund (vgl. [...] Riemer, 2017, S. 174ff.): So zeigt sich, dass diese an Haupt- und Förderschulen überrepräsentiert, in Begabungsförderungsprogrammen z. B. jedoch unterrepräsentiert sind und damit - weitgehend unabhängig von ihren Fähigkeiten - doppelt benachteiligt werden (vgl. z. B. Seitz \& Simon, 2018)“ (Rödel \& Simon, 2019, S. 28).

8 https://www.uni-potsdam.de/de/qlb/das-projekt/sp-3-inklusion-und-heterogenitaet.html, zugegriffen 28.10.2019. 
Eine Parallele zwischen dem Sprachbildungs- und dem Inklusionsdiskurs zeigt sich demnach im Argument der Bildungsgerechtigkeit, das in beiden Diskursen als Ziel vorgebracht wird. So schreibt etwa Lütke (2019), dass ein sprachsensibler Fachunterricht eine Komponente darstelle, die für Kinder und Jugendliche „eine umfassende positive Partizipation im gesellschaftlichen Leben“ und damit insgesamt auch „eine[] größere[] Bildungsgerechtigkeit“ (Rödel \& Lütke, 2019, S. 38) zu schaffen sucht (vgl. auch Becker-Mrotzek, 2016; Dirim \& Mecheril, 2018; Morek \& Heller, 2012). Zumeist wird in diesem Zusammenhang darauf hingewiesen, dass für ,Schulerfolg' die Beherrschung eines bestimmten Registers - oft ,Bildungssprache' genannt vonnöten ist. Von Seiten der Schule wird jedoch häufig vorausgesetzt, dass Schüler*innen dieses Register bereits mitbringen bzw. im Laufe der Schullaufbahn ohne zusätzliche Förderung erwerben; der Registererwerb wird im Unterricht nicht systematisch unterstützt. Dadurch werden diejenigen Lernenden systematisch benachteiligt, die - z.B. aufgrund einer anderen Familiensprache und/oder eines niedrigen sozioökonomischen Status - auf schulische Unterstützung im Registererwerb im höheren Maße als andere Lernenden angewiesen sind. Sprachbildung bzw. „[s]prachbezogene Inklusion“ (Lütke, 2019, S. 43) sollte daher Bedingungen schaffen, in denen „benachteiligten Kindern und Jugendlichen ein[] gleichberechtigte[r] Zugang zu einem breiten sprachlichen und inhaltlichen Erfahrungsspektrum“ (ebd.) geboten wird. Damit sollen zugleich „Erfahrungsräume im sprachlichen Handeln [...] eröffne $[\mathrm{t}]$ und [...] erweiter[t] “ werden (ebd.). Anhand der dargelegten Ausführungen zu den Konzepten Sprachbildung und Inklusion zeigt sich, dass sie keineswegs voneinander getrennt sind, sondern - v.a. in Hinblick auf Zielsetzungen - zentrale Überschneidungen aufweisen. Neben diesen konzeptuellen gilt es jedoch auch handlungspraktische Schnittstellen zu ergründen, die das Unterrichten heterogener Lerngruppen betreffen und sowohl inklusive als auch sprachbildende Maßnahmen beinhalten.

\subsubsection{Sprachsensibel unterrichten $=$ adaptiv unterrichten?}

„In den Schulklassen haben wir es mit einer großen sprachlichen und kulturellen Heterogenität zu tun. Diese sprachliche Heterogenität [...] stellt für die Lehrerinnen und Lehrer eine große Herausforderung dar. Denn wenn ein erheblicher Teil ihrer Schüler [...] die für den Unterricht erforderlichen bildungssprachlichen Fähigkeiten nicht mitbringt, dann erfordert das einen anderen Unterricht “ (Becker-Mrotzek et al., 2012, S. 2).

Auf der Suche nach Unterrichtsformen, die den komplexen Anforderungen an inklusives Lehren und Lernen gerecht werden können, stößt man im gegenwärtigen Diskurs schnell auf adaptives Lehren als ein - wenn auch unterschiedlich konnotiertes - vielversprechendes Konzept im Umgang mit Heterogenität (vgl. z.B. Häcker, 2017; Helmke \& Weinert, 1997; Martschinke, 2015). Dabei ist mit dem Konstrukt adaptiven Unterrichtens ein „noch recht, ungeschliffener Diamant' gefunden“ (Martschinke, 2015, S. 27), den es weiter zu bearbeiten gilt. In der inklusionsorientierten Forschung hat sich vor allem das Konstrukt der adaptiven Lehrkompetenz (Beck et al., 2008; Brühwiler, 2014) als förderlich für adaptives Unterrichten herausgestellt: Es erscheint daher sinnvoll, das Konstrukt mit anderen Konzepten inklusiven Lehrens und Lernens zu vergleichen, weshalb im Folgenden Zusammenhänge zwischen adaptivem und sprachbildnerischem Lehrkräftehandeln erarbeitet werden.

Bereits in den Zielsetzungen von adaptiver Lehrkompetenz und Sprachbildung zeigen sich Parallelen: So wird auch der Begriff der Adaptivität „häufig im Zusammenhang mit Schülerinnen und Schülern verwendet, die besonderer pädagogischer Unterstützung bedürfen, aber in normalen Jahrgangsklassen unterrichtet werden" (Beck et al., 2018, S. 33). Das damit einher- 
gehende Ziel der Bildungsgerechtigkeit steht in engem Zusammenhang mit der sprachbildnerischen Erkenntnis, dass viele Lernende beim Registerausbau gezielte Unterstützung benötigen. Beide Ansätze sind dabei auf die Bereitstellung vielseitiger Lehr-Lern-Angebote ausgerichtet, die ungeachtet möglicher Heterogenitätsdimensionen individuelle Anschlussmöglichkeiten eröffnen. So zielen sowohl Sprachbildung als auch „adaptive Lehrkompetenz [auf, d.V.] die Fähigkeit einer Lehrperson [...] ihren Unterricht so auf die individuellen Voraussetzungen und Möglichkeiten der Lernenden anzupassen, dass möglichst günstige Bedingungen für individuell verstehendes Lernen entstehen und beim Lernen aufrecht erhalten bleiben“ (Beck et al., 2008, S. 47).

Mit Blick auf die Konstruktfacetten der adaptiven Lehrkompetenz (siehe auch Beitrag 2.2 in diesem Band) - Sachkompetenz, diagnostische Kompetenz, didaktische Kompetenz, Klassenführungskompetenz - eröffnet sich zudem ein Schematisierungsraum, der auch auf Ebenen sprachbildnerischen Handelns übertragen werden kann:

Erstens bedarf es für die sprachsensible Aufbereitung von Fachinhalten einer dezidierten adaptiven Sachkompetenz, die es erlaubt, sprachliche Besonderheiten des Faches zu erkennen und für den Unterricht heterogenitätssensibel aufzubereiten. Im Adaptivitätsdiskurs bildet „[r]eichhaltiges, differenziertes und klar strukturiertes Fachwissen [...] eine wesentliche Voraussetzung, damit Lehrpersonen adaptiv auf Stärken und Schwächen von Schülerinnen und Schülern reagieren können" (Brühwiler, 2014, S. 79). Auch für sprachsensibles Unterrichten gilt, dass sich Unterricht grundsätzlich aus einem Zusammenwirken von sprachlichen und fachlichen Anforderungen zusammensetzt - angefangen bei Fachvokabular bis hin zu fachspezifischen Operatoren. Demnach ist im Sinne adaptiven, inklusionssensiblen Unterrichts die Facette des Sachwissens um Fragen zu sprachlichen Anforderungen des Faches zu erweitern, um eine produktive Verknüpfung von (Fach-)Sprache und (Fach-)Unterricht herzustellen.

Zweitens ermöglicht eine ausgeprägte adaptive diagnostische Kompetenz nicht nur die Feststellung von fachlichen und überfachlichen Kompetenzen, sondern auch eine detailliertere Sprachstandsfeststellung. Aus Perspektive der Forschung zur adaptiven Lehrkompetenz kann nur flexibel und individuell auf die Bedürfnisse der Lernenden reagiert werden, wenn eine Lehrperson in der Lage ist, „die Lernvoraussetzungen seitens der Schüler (Vorwissen, allgemeine kognitive Fähigkeiten, fachspezifische Fähigkeiten) zu ermitteln. Je zutreffender die Diagnose ist, desto adaptiver kann das anschließende unterrichtliche Angebot gestaltet und desto angepasster kann das Lernen begleitet und unterstützt werden“ (Beck et al., 2008, S. 42). Diese Erhebung von Voraussetzungen ist um Attribute zu ergänzen, die nicht allein die kognitive Entwicklung betreffen: Auch Interessen, Neigungen, individuelle Hintergründe oder „kulturelle[] und motivationale[] Individualmerkmale“ (Gloystein \& Moser, 2018) sind diagnostisch zu bestimmten. Parallel dazu gilt es, den Sprachstand der Lernenden zu ermitteln, wobei es „keine einfachen Verfahren gibt, mit denen schnelle und allgemein gültige Aussagen zum Sprachstand eines einzelnen Schülers gemacht werden können“ (Beese et al., 2014, S. 35). „Schätzverfahren, Beobachtungen, Profilanalysen und Tests“ (Siems, 2013, S. 2) finden als übliche Methoden zur Sprachstandsfeststellung Anwendung. Ähnlich vielseitig sind die Formen didaktischer Diagnostik (Prengel, 2016), die auch der adaptiven diagnostischen Kompetenz sensu Beck und Kolleg*innen entsprechen und ,im Bereich des formativen Assessment angesiedelt" sind (ebd., S. 51): Hierzu zählen z.B. Unterrichtsbeobachtungen, spontane Äußerungen der Lernenden oder „die mündlichen, schriftlichen, ästhetischen oder enaktiven Mitteilungen und Produkte, die die Kinder in Schulfächern, Lernbereichen und fächerübergreifenden Vorhaben hervorbringen" (ebd., S. 54). 
Drittens ist die für adaptives Unterrichten relevante Facette der adaptiven didaktischen Kompetenz ebenfalls in sprachbildnerischen Konzepten angelegt. Hier hat vor allem das vorweg bestimmte Material sowie die damit verbundene Methodenwahl Einfluss auf fachdidaktische Zielsetzungen (vgl. z.B. Lütke, 2019). So erscheint es zielführend, dass „einzelne Elemente der Sprach- und Kommunikationsförderung aus dem Lerngegenstand entwickelt und umgesetzt“ (Stitzinger, 2019, S. 97) und im Sinne größtmöglicher Barrierefreiheit didaktisiert werden. Die hierfür benötigte Kompetenz umfasst - wie alle Konstruktfacetten adaptiver Lehrkompetenz zum einen adaptive Planungs- und Handlungskompetenzen, die die Unvorhersehbarkeit unterrichtlicher Situationen betreffen; zum anderen sind sprachdidaktische Kompetenzen vonnöten, die sich sowohl auf die Planung als auch die Umsetzung sprachbildender didaktischer Maßnahmen beziehen (vgl. ebd., S. 98). Dabei ist ein geeignetes Maß an kooperativen Lehr-LernFormen anzustreben, die für den inklusiven Unterricht als unverzichtbar gelten (vgl. z.B. Thäle, 2019). „Adaptive Lehrpersonen [...] zeichnen sich [...] nicht einfach dadurch aus, möglichst oft individualisierende Unterrichtsmethoden einzusetzen. Jede Unterrichtsmethode kann einen unterschiedlichen Grad an Adaptivität aufweisen“ (Brühwiler, 2014, S. 85) - gleiches gilt für sprachbildende Konzepte, die zwischen Individualisierung und Kooperation changieren, um unterschiedliche Anschlussstellen zu schaffen und verschiedene Lernwege zu eröffnen.

Die vierte Facette adaptiver Lehrkompetenz betrifft den Bereich der adaptiven Klassenführungskompetenz. Hier sind weniger deutliche Parallelen zum Bereich der Sprachbildung zu ziehen; vielmehr ist adaptive Klassenführungskompetenz als Bedingung für sprachbildendes unterrichtliches Handeln geltend zu machen: „Mit der Kompetenz, eine Klasse zu führen, schafft eine Lehrperson die Grundvoraussetzungen und setzt die Rahmenbedingungen, damit systematisches Lernen im Unterricht überhaupt möglich ist" (Beck et al., 2008, S. 46). Dabei sind Klarheit - auch in der Sprache - im Hinblick auf die geplante Unterrichtseinheit, die Gewährleistung eines reibungslosen Ablaufs und ein schüler*innenaktivierender Unterricht genauso relevant wie die Einhaltung von Regeln und Routinen, produktives Feedback, Motivierung sowie eine grundsätzlich wertschätzende Unterrichtskultur (vgl. z.B. Haag, 2018). Ein Blick beispielsweise in die Transkription von Unterrichtsdialogen im Rahmen des Sprachbildungsprojektes FörMig (Quehl \& Trapp, 2013) bestätigt diesen Ansatz auch aus sprachbildnerischer Perspektive: Die dort handelnde Lehrkraft adressiert die Schüler*innen mit der notwendigen Deutlichkeit und Klarheit in sowohl Sprache als auch Zielvorgabe und gibt dabei aktivierende, wertschätzende Rückmeldungen (vgl. ebd., S. 64-76).

Die dargestellten Konstruktfacetten adaptiver Lehrkompetenz betreffen sowohl die Vorbereitungs- als auch die Handlungsebene bzw. die Makro- und die Mikroebene des Unterrichtshandelns. Während im Rahmen adaptiver Lehrkompetenz die Makroebene vor allem die Planung und Vorbereitung hinsichtlich der „Inhalte, Methoden, Medien, Materialien, Sozialformen und Lernzeiten“ (Schmitz, 2017) betrifft, wird die Mikroebene anhand der „direkten und individuellen Lehrer-Schüler-Interaktion“ definiert, wobei das „Hauptaugenmerk [...] auf individueller Unterstützung und Rückmeldung (im Sinne eines Feedbacks)“ liegt (Martschinke, 2015, S. 24). Eine analoge Unterteilung in Makro- und Mikroebene wird im Bereich der Sprachbildung z.B. im Scaffolding-Konzept vorgenommen: Auf der Makroebene werden das Vorwissen, die Materialauswahl, eine Sequenzierung der Lernaufgaben und die Bestimmung von Lern- und Arbeitsformen sowie Medien in der Planung berücksichtigt. Hierfür spielen sowohl vermittelnde Brückentexte, die den Zugang zu komplexeren Sprachstrukturen schaffen, als auch Texte, die als Lernanreiz unmittelbar über dem sprachlichen Kompetenzniveau der Lernenden liegen, eine maßgebliche Rolle. Im Micro-Scaffolding werden unterstützende Sprachhandlungen in der 
Interaktion vollzogen - etwa durch Reduzierung des Tempos, Gewährung unterschiedlicher Zeitressourcen oder einer Variation der Interaktionsformen und -muster (vgl. Kniffka, 2010). Der Vergleich der Konzepte Sprachbildung und adaptives Unterrichten zeigt, dass die einzelnen Facetten des Konstrukts adaptive Lehrkompetenz zahlreiche Bezüge zu sprachbildenden Ansätzen aufweisen. Werden diese synergetisch betrachtet, bilden sich neue Perspektiven und Handlungsformen für die inklusionssensible Unterrichtsgestaltung heraus: sowohl kontextund situationsspezifisch im Sinne adaptiven Agierens, als auch orientiert an den Kompetenzen der Schüler*innen, etwa bezogen auf die Heterogenitätsdimension Sprache.

\subsubsection{Fazit}

Neue Herausforderungen im Lehrkräftehandeln erfordern neue Maßnahmen für die Lehrkräftebildung. Besonders für das Unterrichten heterogener Lerngruppen können verschiedene Querschnittsdisziplinen und -konzepte ausgemacht werden, die zwar unterschiedliche Schwerpunkte setzen, jedoch in direktem Zusammenhang zueinander stehen. Deshalb sollten diese Ansätze nicht unabhängig voneinander, sondern in produktiver Wechselwirkung miteinander untersucht und sowohl für den Schulunterricht als auch für die universitäre Lehrkräftebildung aufbereitet werden. Als übergeordneter Themenbereich ist zunächst das Thema Inklusion herauszustellen, das andere (für heterogenitätssensible Formate relevante) Bereiche, etwa den der Sprachbildung, mit einschließen kann. Ziel von sowohl Inklusion als auch Sprachbildung ist die Überwindung von Barrieren und die Herstellung umfassender Partizipation und Bildungsgerechtigkeit, sodass allen Schüler*innen die Entwicklung individueller Kompetenzen gewährt wird. Als aussichtsreiches Konzept für die Erreichung dieses Ziels gilt das Konstrukt der adaptiven Lehrkompetenz, das in den Konstruktfacetten adaptive Sach-, diagnostische, didaktische und Klassenführungskompetenz vielversprechende Überschneidungspunkte mit sprachbildnerischen Ansätzen aufweist. Neben hier zu knüpfenden Synergien, die auf einen produktiven, wertschätzenden Umgang mit Heterogenität abzielen und inklusionsorientierte Handlungsspielräume für (zukünftige) Lehrkräfte eröffnen, verspricht die Verbindung beider Konzepte auch neue theoretische Ansätze auf Forschungsebene: So zeigt dieser erste explorative Vergleich, dass Sprachbildung und adaptive Lehrkompetenz Ähnlichkeiten auf der systemischen Ebene aufweisen, deren gemeinsame Behandlung fruchtbar für Forschung und Lehre erscheint - etwa im Rahmen von Untersuchungen zu Fragen inklusionssensiblen Unterrichtens auf sowohl der Makro- als auch der Mikroebene. Die hier vorgestellte Systematisierung soll als erster Versuch zur Bestimmung von Gemeinsamkeiten und Unterschieden einzelner Ansätze gelten, um neue theoretische und praktische Entwicklungsfelder zu eröffnen, die sich aus der Verschränkung unterschiedlicher Diskurse mit ähnlichen Zielsetzungen ergeben.

\section{Literatur}

Baumann, B. (2017). Sprachförderung und Deutsch als Zweitsprache in der Lehrerbildung - ein deutschlandweiter Überblick. In M. Becker-Mrotzek, P. Rosenberg, C. Schroeder \& A.Witte (Hrsg.), Deutsch als Zweitsprache in der Lehrerbildung (Sprachliche Bildung, Bd. 2, S. 9-26). Münster: Waxmann.

Beck, E., Baer, M., Guldimann, T., Bischoff, S., Brühwiler, C. \& Müller, P. (Hrsg.). (2008). Adaptive Lehrkompetenz. Analyse und Struktur, Veränderbarkeit und Wirkung handlungssteuernden Lehrerwissens. Münster: Waxmann.

Becker-Mrotzek, M. (2016). Inklusive sprachliche Bildung - Perspektiven aus der Sicht der Sprachdidaktik. In D. Gebele \& A.L. Zepter (Hrsg.), Inklusion. Sprachdidaktische Perspektiven: Theorie, Empirie, Praxis (S. 47-56). Duisburg: Gilles \& Francke Verlag. http://koebes.phil-fak.uni-koeln.de/sites/koebes/user_upload/KoeBes_16_ Gebele_Zepter.pdf. Zugegriffen 01.08.2019. 
Becker-Mrotzek, M., Hentschel, B., Hippmann, K. \& Linnemann, M. (2012). Sprachforderung in deutschen Schulen die Sicht der Lehrerinnen und Lehrer. Ergebnisse einer Umfrage unter Lehrerinnen und Lehrern, durchgeführt von IPSOS (Hamburg) im Auftrag des Mercatorinstituts für Sprachförderung und Deutsch als Zweitsprache. Köln: Universität zu Köln. https://www.mercator-institut-sprachfoerderung.de/fileadmin/user_upload/Lehrerumfrage_Langfassung_final_30_05_03.pdf. Zugegriffen 05.08.2019.

Beese, M., Benholz, C., Chlosta, C., Gürsoy, E., Hinrichs, B., Niederhaus, C. \& Oleschko, S. (2014). Sprachbildung in allen Fächern. München: Klett-Langenscheidt.

Bergemann, W. (2015). Von der inklusiven Schule würden mehrsprachige Kinder profitieren. Interview mit Prof. Dr. Claudia Riemer, Mercatorinstitut für Sprachförderung und Deutsch als Zweitsprache. https://www.mercator-institutsprachfoerderung.de/de/themenportal/thema/von-der-inklusiven-schule-wuerden-mehrsprachige-kinder-profitieren/. Zugegriffen 05.08.2019.

Berkemeier, A. \& Kaltenbacher, E. (2014). Zum Verhältnis von Zweitsprachförderung und (Deutsch-)Unterricht. In S. Trumpa, S. Seifried, E. Franz \& T. Klauß (Hrsg.), Inklusive Bildung. Erkenntnisse und Konzepte aus Fachdidaktik und Sonderpädagogik (S. 292-307). Weinheim: Beltz.

Brühwiler, C. (2014). Adaptive Lehrkompetenz und schulisches Lernen. Effekte handlungssteuernder Kognitionen von Lehrpersonen auf Unterrichtsprozesse und Lernergebnisse der Schülerinnen und Schüler. Münster: Waxmann.

Chilla, S. (2017). Sprachliche Bildung und Schulerfolg. In M. Gercke, S. Opalinski \& T. Thonagel (Hrsg.), Inklusive Bildung und gesellschaftliche Exklusion. Zusammenhänge - Widersprüche - Konsequenzen (S. 123-136). Wiesbaden: Springer VS. doi: 10.1007/978-3-658-17084-4_8

Daveri, L. \& Weilbrenner, M. (2013). Praxisportrait: Inklusion durch sprachliche Bildung und Elternbildung am Beispiel des Programms Griffbereit. In W. Stange (Hrsg.), Erziehungs- und Bildungspartnerschaften. Praxisbuch zur Elternarbeit (S. 127-132). Wiesbaden: Springer VS. doi: 10.1007/978-3-531-94295-7_12

Dirim, İ. \& Mecheril, P. (Hrsg.). (2018). Heterogenität, Sprache(n), Bildung. Eine differenz-und diskriminierungstheoretische Einführung. Bad Heilbrunn: Klinkhardt.

Flieger, P. (2017). Partizipation. In K. Ziemen (Hrsg.), Lexikon Inklusion (S. 179-180). Göttingen: Vandenhoeck \& Ruprecht. doi: 10.13109/9783666701870

Frohn, J. (2019). Individuelle Kompetenzentwicklung. In J. Frohn, E. Brodesser, V. Moser \& D. Pech (Hrsg.), Inklusives Lehren und Lernen. Allgemein- und fachdidaktische Grundlagen (S. 75-78). Bad Heilbrunn: Klinkhardt.

Haag, L. (2018). Kernkompetenz Klassenfübrung. Bad Heilbrunn: Klinkhardt.

Häcker, T. (2017). Individualisierter Unterricht. In T. Bohl, J. Budde \& M. Rieger-Ladich (Hrsg.), Umgang mit Heterogenität in Schule und Unterricht. Grundlagentheoretische Beiträge, empirische Befunde und didaktische Reflexionen (S. 275-290). Bad Heilbrunn: Klinkhardt.

Gloystein, D. \& Moser, V. (2019). Ausgangslage. In J. Frohn, E.Brodesser, V. Moser \& D. Pech (Hrsg.), Inklusives Lehren und Lernen. Allgemein- und fachdidaktische Grundlagen (S. 65-67). Bad Heilbrunn: Klinkhardt.

Helmke, A. \& Weinert, F.E. (1997). Bedingungsfaktoren schulischer Leistungen. In F.E. Weinert (Hrsg.), Enzyklopädie der Psychologie, Psychologie der Schule und des Unterrichts (Bd. 3, S. 71-76). Göttingen: Hogrefe.

Kniffka G. (2010). Scaffolding. In Kompetenzzentrum ProDaZ. https://www.uni-due.de/imperia/md/content/prodaz/scaffolding.pdf. Zugegriffen 05.08.2019.

König, A. \& Friederich, T. (Hrsg.). (2015). Inklusion durch sprachliche Bildung. Neue Herausforderungen im Bildungssystem. Weinheim: Beltz.

Lütke, B. (2019). Sprachsensibler Fachunterricht im Spiegel von Sprachbildung und Inklusion. In L. Rödel \& T. Simon (Hrsg.), Inklusive Sprach(en)bildung - Ein interdisziplinärer Blick auf das Verhältnis von Inklusion und Sprachbildung (S. 38-48). Bad Heilbrunn: Klinkhardt.

Martschinke, S. (2015). Facetten adaptiven Unterrichts aus der Sicht der Unterrichtsforschung. In K. Liebers, B. Landwehr, A. Marquardt \& K. Schlotter (Hrsg.), Lernprozessbegleitung und adaptives Lernen in der Grundschule (S. 15-32). Wiesbaden: Springer VS. doi: 10.1007/978-3-658-11346-9_2

Morek, M. \& Heller, V. (2012). Bildungssprache - Kommunikative, epistemische, soziale und interaktive Aspekte ihres Gebrauchs. Zeitschrift für angewandte Linguistik 57(1), S. 67-101. doi:10.1515/zfal-2012-0011

Prengel, A. (2016). Didaktische Diagnostik als Element alltäglicher Lehrerarbeit. „formatives Assessment im inklusiven Unterricht“. In B. Amrhein (Hrsg.), Diagnostik im Kontext inklusiver Bildung. Theorien, Ambivalenzen, Akteure, Konzepte (S. 49-63). Bad Heilbrunn: Klinkhardt.

Professional School of Education. (2019). Professional School of Education (PSE). https://pse.hu-berlin.de/de/aktuelles/standardseite. Zugegriffen 23.08.2019.

Quehl, T. \& Trapp, U. (2013). Sprachbildung im Sachunterricht der Grundschule. Mit dem Scaffolding-Konzept unterwegs zur Bildungssprache (FörMig-Material, Bd. 4). Münster: Waxmann.

Riemer, C. (2017). DaZ und Inklusion - Gemeinsamkeiten und Unterschiede. Ein fachpolitischer Positionierungsversuch aus der Perspektive des Fachs DaF/DaZ. In M. Becker-Mrotzek, P. Rosenberg, C. Schroeder \& A. Witte (Hrsg.), Deutsch als Zweitsprache in der Lehrerbildung (Sprachliche Bildung, Bd. 2, S. 171-186). Münster: Waxmann. 
Rödel, L. \& Lütke, B. (2019). Sprachbildung im inklusiven Fachunterricht. In J. Frohn, E. Brodesser, V. Moser \& D. Pech (Hrsg.), Inklusives Lehren und Lernen. Allgemein- und fachdidaktische Grundlagen (S. 81-88). Bad Heilbrunn: Klinkhardt.

Rödel, L. \& Simon, T. (2018). Zum Verhältnis von Inklusion und Sprachbildung - Einblicke, offene Fragen, Forschungsperspektiven. Zeitschrift für Inklusion. https:/www.inklusion-online.net/index.php/inklusion-online/ article/view/484. Zugegriffen 11.08.2019.

Rödel, L. \& Simon, T. (2019). Inklusive Sprachbildung - Eine Einladung zum transdisziplinären Dialog. In L. Rödel \& T. Simon (Hrsg.), Inklusive Sprach(en)bildung - Ein interdisziplinärer Blick auf das Verhältnis von Inklusion und Sprachbildung (S. 24-37). Bad Heilbrunn: Klinkhardt.

Schmitz, L. (2017). Adaptive Lehrkompetenz. In J. Frohn (Hrsg.), FDQI-HU-Glossar. Berlin: Humboldt-Universität zu Berlin. https://pse.hu-berlin.de/de/forschung-und-lehre/projekte/fdqi-hu/Inklusionsglossar/copy_of_adaptive-lehrkompetenz. Zugegriffen 05.11.2019.

Settinieri, J. (2017). Aus Deutsch für Ausländer wird Deutsch als Fremdsprache wird Deutsch als Zweitsprache wird Durchgängige Sprachbildung... wird Inklusion? In E. Burwitz-Melzer, F.G. Königs, C. Riemer \& L. Schmelter (Hrsg.), Inklusion, Diversität und das Lehren und Lernen fremder Sprachen. Arbeitspapiere der 37. Frühjahrskonferenz zur Erforschung des Fremdsprachenunterrichts (S. 306-313). Tübingen: Narr Francke Attempto.

Siems, M. (2013). Verfahren der Sprachstandsfeststellung für Schülerinnen und Schüler mit Deutsch als Zweitsprache. https://www.uni-due.de/imperia/md/content/prodaz/sprach-standsfeststellungsverfahren_siems.pdf. Zugegriffen 29.07.2019.

Simon, T. (2019). Zum Inklusionsverständnis in FDQI-HU. In J. Frohn, E. Brodesser, V. Moser \& D. Pech (Hrsg.), Inklusives Lehren und Lernen. Allgemein- und fachdidaktische Grundlagen (S. 21-27). Bad Heilbrunn: Klinkhardt.

Simon, T. \& Pech, D. (2019). Partizipation. In J. Frohn, E. Brodesser, V. Moser \& D. Pech (Hrsg.), Inklusives Lehren und Lernen. Allgemein- und fachdidaktische Grundlagen (S. 40-41). Bad Heilbrunn: Klinkhardt.

Stitzinger, U. (2019). Pädagogik bei Beeinträchtigungen der Sprache und Sprachtherapie inklusiv verortet. In L. Rödel \& T. Simon (Hrsg.), Inklusive Sprach(en)bildung - Ein interdisziplinärer Blick auf das Verhältnis von Inklusion und Sprachbildung (S. 87-101). Bad Heilbrunn: Klinkhardt.

Thäle, A. (2019). Kooperation. In J. Frohn, E. Brodesser, V. Moser \& D. Pech (Hrsg.), Inklusives Lehren und Lernen. Allgemein- und fachdidaktische Grundlagen (S. 50-52). Bad Heilbrunn: Klinkhardt.

Tschernig, K. \& Vo Thi, M.H. (2017). Die Diversitätsdimension Sprache als Schlüsselqualifikation im Fachunterricht: diskursbestimmende Konzepte und inklusionsspezifische Paradigmen. Zeitschrift für Inklusion (3). https://www. inklusion-online.net/index.php/inklusion-online/article/view/449/333. Zugegriffen 01.08.2019. 